\title{
On the necessity of identifying the true parameter in adaptive LQ control
}

\author{
J.W. POLDERMAN \\ Centre for Mathematics and Computer Science, P.O. Box 4079, 1009 AB Amsterdam, The Netherlands
}

Received 3 February 1986

Revised 18 April 1986

Abstract: In adaptive control problems one may drop the requirement of identifying the true system in order to simplify the problem of control. It will be shown that in the adaptive LQ control problem this does not at all lead to an easier problem.

AMS Subject Classification: $93 \mathrm{C} 40$.

Keywords: Adaptive LQ control, Closed-loop identification, Certainty equivalence.

\section{Introduction}

A very popular approach to the problem of controlling an unknown plant adaptively is the use of the so called certainty equivalence principle. Based on the observations upto time $t$ one makes an estimation of the characteristics of the plant, and then, as far as the control to be applied is concerned, one acts as if this estimation represents the real system. Of course certainty equivalence does not have to hold, but it is just imposed because of the simple structure of the resulting control scheme.

This method causes certain identifiability problems. First of all there is the problem of excitation: the signals going into the system to be rich enough in order to reveal the relevant characteristics of the plant.

A second difficulty one is faced with is the fact that identification takes place in closed loop which makes it hard or even impossible to identify the real system. This short note deals with the second problem only. This will be done in the following situation. The real system is assumed to be deterministic, linear, time invariant and with known number of inputs and states. Moreover the state is assumed to be observed. The control objective will be the minimization of a quadratic cost functional. If the parameters of the system were known this objective could be achieved; in the adaptive situation we can only hope to identify the optimal control law asymptotically.

Identification of the optimal closed-loop system may seem easier to achieve than identification of the true open-loop system. For it can be expected that there are a lot of models that give rise to the same optimal control law, and hence identification of the true system is not required. It will be enough that the sequence of control laws corresponding to the parameter estimates converges to the true optimal one.

We will show however that among those parameters which correspond to the true optimal control law, the true one is the only one that can be identified in closed loop. In [2] this result was already obtained for the case that the state space is one dimensional. Since our. considerations hold for every estimation scheme, we do not refer to any such scheme at all.

In Section 2 we will give an exact description of the model class and the control objective. Two subsets of the model class will be introduced to formalize the result stated above. Section 3 is devoted to the proof of the claim. Both the discrete and the continuous time case will be covered. 


\section{Problem formulation}

Consider the following set:

$$
E_{C}:=\left\{(A, B) \in \mathbb{R}^{n \times n} \times \mathbb{R}^{n \times m} \mid(A, B, C) \text { minimal and } B \text { of full column rank }\right\},
$$

where $C \in \mathbb{R}^{p \times n}$ is fixed and given. The numbers $n$ and $m$ are assumed to be known. An element $(A, B) \in E_{C}$ represents one of the following systems (depending on whether one is working in discrete or continuous time):

$$
\begin{array}{ll}
\dot{x}=A x_{1}+B u_{1}, \quad x_{0} \in \mathbb{R}^{n} & \text { (continuous-time case) } \\
x_{k+1}=A x_{k}+B u_{k}, \quad x_{0} \in \mathbb{R} & \text { (discrete-time case). }
\end{array}
$$

Suppose the control objective is the minimization of the following expressions ${ }^{1}$ :

$$
\begin{aligned}
& J_{\mathrm{c}}=\int_{0}^{\infty}\left(x_{l}^{\mathrm{T}} Q x_{l}+u_{\imath}^{\mathrm{T}} R u_{l}\right) \mathrm{d} t, \\
& J_{\mathrm{d}}=\sum_{k=0}^{\infty}\left(x_{k}^{\mathrm{T}} Q x_{k}+u_{k}^{\mathrm{T}} R u_{k}\right),
\end{aligned}
$$

where $Q=C^{\mathrm{T}} C$, and $R=R^{\mathrm{T}}>0$.

The solutions of these problems are well known (see [1]), and are given by

$$
u_{t}=F_{\mathrm{c}}(A, B) x_{t}, \quad u_{k}=F_{\mathrm{d}}(A, B) x_{k},
$$

where

$$
\begin{aligned}
& F_{\mathrm{c}}(A, B)=-R^{-1} B^{\mathrm{T}} K_{\mathrm{c}}, \\
& F_{\mathrm{d}}(A, B)=-\left(B^{\mathrm{T}} K_{\mathrm{d}} B+R\right)^{-1} B^{\mathrm{T}} K_{\mathrm{d}} A,
\end{aligned}
$$

and $K_{\mathrm{c}}$ and $K_{\mathrm{d}}$ are the unique symmetric positive definite solutions of

$$
\begin{aligned}
& A^{\mathrm{T}} K+K A-K B R^{-1} B^{\mathrm{T}} K+Q=0, \\
& K-A^{\mathrm{T}} K A+A^{\mathrm{T}} K B\left(B^{\mathrm{T}} K B+R\right)^{-1} B^{\mathrm{T}} K A-Q=0,
\end{aligned}
$$

respectively. Moreover the optimal costs are given by

$$
x_{0}^{\mathrm{T}} K_{\mathrm{c}} x_{0} \text { and } x_{0}^{\mathrm{T}} K_{\mathrm{d}} x_{0}
$$

where $x_{0}$ is the initial state of the system.

Let the real system be represented by the (unknown) pair $\left(A_{0}, B_{0}\right) \in E_{C}$. Since we do not know ( $\left.A_{0}, B_{0}\right)$, we reformulate the control objective as the (asymptotic) identification of $F\left(A_{0}, B_{0}\right)$. Hence the sequence of parameter estimates should most desirably converge to the following subset of the parameter space:

$$
H_{\mathrm{c}}:=\left\{(A, B) \in E_{C} \mid F_{\mathrm{c}}(A, B)=F_{\mathrm{c}}\left(A_{0}, B_{0}\right)\right\} .
$$

$H$ can be seen as the set of desirable limit points of the estimation scheme. Since identification takes place in closed loop, the set of possible points is given by

$$
G_{\mathrm{c}}:=\left\{(A, B) \in E_{C} \mid A+B F_{\mathrm{c}}(A ; B)=A_{0}+B_{0} F_{\mathrm{c}}(A, B)\right\} \text {. }
$$

$G_{\mathrm{d}}$ and $H_{\mathrm{d}}$ are defined similarly.

${ }^{1}$ Subscripts $\mathrm{c}$ and $\mathrm{d}$ refer to continuous and discrete time respectively. 
The reader is referred to [3] for a more elaborate discussion of the interpretation of $G$ and $H$. Also it is proved there that $G$ and $H$ are $C^{\omega}$-manifolds of dimensions $m \times n$ and $n \times n$ respectively.

\section{The intersection of $G$ and $H$}

The interpretation of the intersection of $G$ and $H$ is that it consists of those parameters that correspond to the optimal control law and which are also identifiable in closed loop. Unfortunately it will turn out that generically $G \cap H$ contains only the true system. This result is easy to derive in the continuous-time case, whereas for the discrete-time case we will need some lemmata.

The following lemma holds for both the discrete and the continuous time case.

Lemma 3.1. Let $(A, B) \in G_{\mathrm{d}}\left(\right.$ or $\left.G_{\mathrm{c}}\right)$, denote the solution of (2.9) (or (2.8)) by $K$ and let $K_{0}$ be the solution of (2.9) (or (2.8)) with $(A, B)$ replaced by $\left(A_{0}, B_{0}\right)$. Then $K \geqslant K_{0}$.

Proof. Let $x_{0} \in \mathbb{R}^{n}$; the optimal costs for the system $(A, B)$, starting in $x_{0}$, are $x_{0}^{\mathrm{T}} K x_{0}$, the optimal costs for $\left(A_{0}, B_{0}\right)$ are $x_{0}^{\mathrm{T}} K_{0} x_{0}$. The real costs incurred when the feedback $F_{\mathrm{d}}(A, B)$ is applied to the system $\left(A_{0}, B_{0}\right)$ are equal to the optimal costs of the system $(A, B)$, since $(A, B) \in G_{\mathrm{d}}$ and hence both the state and input trajectory of $A+B F(A, B)$ and $A_{0}+B_{0} F(A, B)$ are equal. However, for $\left(A_{0}, B_{0}\right), F_{\mathrm{d}}(A, B)$ can do no better than $F_{\mathrm{d}}\left(A_{0}, B_{0}\right)$. Hence $x_{0}^{\mathrm{T}} K x_{0} \geqslant x_{0}^{\mathrm{T}} K_{0} x_{0}$, since $x_{0}$ was arbitrary it follows that $K \geqslant K_{0}$.

Corollary 3.2. If $(A, B) \in G_{\mathrm{d}} \cap H_{\mathrm{d}}\left(\right.$ or $\left.G_{\mathrm{c}} \cap H_{\mathrm{c}}\right)$, then $K=K_{0}$.

Proof. Since $(A, B) \in G_{\mathrm{d}}$, we have $A+B F_{\mathrm{d}}(A, B)=A_{0}+B_{0} F_{\mathrm{d}}(A, B)$, which by Lemma 3.1 implies that $K \geqslant K_{0}$. On the other hand, since $(A, B) \in H_{\mathrm{d}}$, we also have $A_{0}+B_{0} F_{\mathrm{d}}\left(A_{0}, B_{0}\right)=A+B F_{\mathrm{d}}\left(A_{0}, B_{0}\right)$. We can apply Lemma 3.1 once again, now with $\left(A_{0}, B_{0}\right)$ and $(A, B)$ interchanged, showing that $K_{0} \geqslant K$.

Let us now consider the continuous-time case; as a direct consequence of Corollary 3.2 we have:

Theorem 3.3. $H_{\mathrm{c}} \cap G_{\mathrm{c}}=\left\{\left(A_{0}, B_{0}\right)\right\}$.

Proof. It is trivial to see that the right-hand side is contained in the left-hand side, and the other inclusion is almost trivial:

$$
\begin{aligned}
& (A, B) \in G_{\mathrm{c}} \cap H_{\mathrm{c}} \Rightarrow K=K_{0} \quad \text { (by Corollary 3.2), } \\
& (A, B) \in H_{\mathrm{c}} \Rightarrow B^{\mathrm{T}} K=B_{0}^{\mathrm{T}} K_{0} ;
\end{aligned}
$$

(3.1) and (3.2) together give $B=B_{0}$; substituting this in (2.12) gives $A=A_{0}$.

If we impose an additional condition on $A_{0}$, then the same result can be obtained for the discrete-time case. However the proof is somewhat more involved, and therefore we will divide it into several parts.

Lemma 3.4. For every $(A, B) \in E_{C}$ one has $\operatorname{Ker}\left(A+B F_{\mathrm{d}}(A, B)\right)=\operatorname{Ker} A$.

Proof. Suppose $x_{0} \in \operatorname{Ker}\left(A+B F_{\mathrm{d}}(A, B)\right)$; then $x_{k}=0$ and $u_{k}=0$, for all $k \geqslant 1$. Hence

$$
\begin{aligned}
x_{0}^{\mathrm{T}} K_{\mathrm{d}} x_{0} & =x_{0}^{\mathrm{T}} Q x_{0}+u_{0}^{\mathrm{T}} R u_{0} \quad(\text { by }(2.4) \text { and }(2.10)) \\
& =x_{0}^{\mathrm{T}}\left(Q+F_{\mathrm{d}}(A, B)^{\mathrm{T}} R F_{\mathrm{d}}(A, B)\right) x_{0} \quad(\text { by }(2.5)) \\
& \left.=x_{0}^{\mathrm{T}}\left(K_{\mathrm{d}}-A^{\mathrm{T}} K_{\mathrm{d}}\left(A+B F_{\mathrm{d}}(A, B)\right)+F_{\mathrm{d}}(A, B)^{\mathrm{T}} R F_{\mathrm{d}}(A, B)\right) x_{0} \quad \text { (by }(2.7) \text { and }(2.9)\right) .
\end{aligned}
$$


This implies that $x_{0}^{\mathrm{T}} F_{\mathrm{d}}(A, B)^{\mathrm{\top}} R F_{\mathrm{d}}(A, B) x_{0}=0$ and thus that $F_{\mathrm{d}}(A, B) x_{0}=0$. Together with $(A+$ $\left.B F_{\mathrm{d}}(A, B)\right) x_{0}=0$ this gives $A x_{0}=0$.

Suppose on the other hand that $A x_{0}=0$; then also $F_{\mathrm{d}}(A, B) x_{0}=0$ (by (2.7)) and thus $(A+$ $\left.B F_{\mathrm{d}}(A, B)\right) x_{0}=0$.

Since $\left(A_{0}, B_{0}\right)$ is controllable, rank $A_{0} \geqslant n-m$. By Lemma 3.4 we may conclude that $\operatorname{rank}\left(A_{0}+\right.$ $\left.B_{0} F_{\mathrm{d}}\left(A_{0}, B_{0}\right)\right)^{\mathrm{T}} K_{0} \geqslant n-m$. Let $r:=\operatorname{dim} \operatorname{Ker}\left(A_{0}+B_{0} F_{\mathrm{d}}\left(A_{0}, B_{0}\right)\right)^{\mathrm{T}} K_{0}$. Then $r \leqslant m$, hence we can give:

Definition 3.5. Define $\bar{B} \in \mathbb{R}^{n \times m}$ by $\bar{B}=\left[\bar{b}_{1}, \ldots, \bar{b}_{r}, 0, \ldots, 0\right]$, where $\bar{b}_{1}, \ldots, \bar{b}_{r}$ is a basis of $\operatorname{Ker}\left(A_{0}+\right.$ $\left.B_{0} F_{\mathrm{d}}\left(A_{0}, B_{0}\right)\right)^{\mathrm{T}} K_{0}$.

Theorem 3.6. $G_{\mathrm{d}} \cap H_{\mathrm{d}} \subseteq\left\{\left(A_{0}-\bar{B} \Lambda F_{0}, B_{0}+\bar{B} \Lambda\right) \mid \Lambda \in \mathbb{R}^{m \times m}\right\} \cap E_{C}$.

Proof. Denote by $F_{0}$ the true optimal control law and with $K$ the solution of (2.9). Then

$$
\begin{aligned}
& (A, B) \in G_{\mathrm{d}} \Rightarrow A=A_{0}+\left(B_{0}-B\right) F_{0}, \\
& (A, B) \in H_{\mathrm{d}} \Rightarrow\left(B^{\mathrm{T}} K B+R\right)^{-1} B^{\mathrm{T}} K A=-F_{0} .
\end{aligned}
$$

Substituting (3.3) in (3.4) gives

$$
B^{\mathrm{T}} K\left(A_{0}+\left(B_{0}-B\right) F_{0}\right)=-\left(B^{\mathrm{T}} K B+R\right) F_{0}
$$

which implies

$$
B^{\mathrm{T}} K\left(A_{0}+B_{0} F_{0}\right)=-R F_{0} .
$$

Now, consider (3.5) as an equation in $B$. A particular solution of (3.5) is by construction $B=B_{0}$. Every solution of the homogeneous equation can be written as $\bar{B} \Lambda$ for some $\Lambda \in \mathbb{R}^{m \times m}$. This shows that the general solution of (3.5) is

$$
B=B_{0}+\bar{B} \Lambda \text {. }
$$

Substituting (3.6) in (3.3) gives the statement.

Corollary 3.7. In the generic case where $A_{0}$ is non-singular we have

$$
G_{\mathrm{d}} \cap H_{\mathrm{d}}=\left\{\left(A_{0}, B_{0}\right)\right\} \text {. }
$$

Proof. If $A_{0}$ is non-singular, then $r=0$, which implies that $\bar{B}=0$.

\section{Conclusion}

The purpose of an adaptive controller may include, besides the optimal control objective, the identification of the true system. If we drop the identification requirement, then we could expect (at least at first sight) and easier task to fulfill. This is confirmed by the fact that there is an $(n \times n)$-dimensional submanifold of the parameter space of parameter values that all give the desired closed-loop behaviour, namely the set $H$. Although $G$ has been called the set of possible limit points of an estimation scheme, it is not claimed that there exists any such scheme that will give convergence of the sequence of parameter estimates to $G$ (although for $n=1$ it does). However $G$ is pointwise invariant under any 'estimation in closed loop' procedure based on the observed state, and from any point outside $G$ we may eventually be thrown of. So from Theorem 3.3 and Corollary 3.7 we may conclude that in fact we have to identify the true system in order to achieve optimal closed-loop behaviour. 


\section{References}

[1] H. Kwakernaak and R. Sivan, Linear Optimal Control Systems (John Wiley \& Sons, New York, 1972).

[2] W. Lin, P.R. Kumar, T.I. Seidman, Will the self-tuning approach work for general cost criteria? Systems Control Lett. 6 (1985) $77-86$.

[3] J.W. Polderman, A note on the structure of two subsets of the parameter space in adaptive control problems, Systems Control Lett. 7 (1985) 25-34. 